Proceedings of the 16th Czech and Slovak Conference on Magnetism, Košice, Slovakia, June 13-17, 2016

\title{
Characterization of Carbon Nanotubes
}

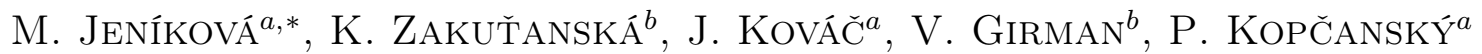 \\ AND N. TOMǍ̌OVIČOVÁ $a$ \\ ${ }^{a}$ Institute of Experimental Physics, SAS, Watsonova 47, 04001 Košice, Slovakia \\ ${ }^{b}$ Institute of Physics, Faculty of Sciences, P.J. Šafárik University, Park Angelinum 9, 04001 Košice, Slovakia

\begin{abstract}
The aim of the presented work was to characterize single-walled carbon nanotubes as well as multi-walled carbon nanotubes by transmission electron microscopy, the Raman spectroscopy and magnetization measurements to obtain information about their size, structure, and magnetic properties. We show that having different carbon nanotubes one can easily distinguish the single-wall or multi-wall carbon nanotubes and determine their quality. The obtained results show that carbon nanotubes can be diamagnetic or ferromagnetic depending on their structural parameters.
\end{abstract}

DOI: 10.12693/APhysPolA.131.952

PACS/topics: 61.48.De, 74.25.nd, 68.37.Lp, 75.75.--c

\section{Introduction}

Interest in study of binary mixtures of nematic liquid crystals and carbon nanotubes (CNTs) is motivated by their common high anisotropic physical properties [1]. CNTs are exceptionally anisometric particles with diameters of the order of $\mathrm{nm}$, but lengths ranging from $\mu \mathrm{m}$ to $\mathrm{cm}[2]$. Each CNT is such as a graphene sheet rolled up into tubular morphology (single walled CNTs) or several such tubes arranged concentrically (multiwalled CNTs). They can have either a paramagnetic, ferromagnetic or diamagnetic response to an applied magnetic field depending on their diameter, chirality and the Fermi energy level. In general, defects considerably affect the electronic, mechanical and chemical properties of CNTs [3]. However, structural defects on the outer surface of CNTs can also be beneficial when it comes to hybridizing them with metal oxides or other materials. Defects can serve as reaction centers for functionalization and as nucleation sites for crystal growth, influencing both crystallization (i.e. crystal structure, crystal defects) and growth (i.e. size, morphology) of the coating material. Control of CNTs orientation becomes very important for their application in new materials. Among the methods that have been proposed are magnetic and electric field alignment. One from the promising methods is their dispersion in liquid crystal. To prepare stable colloids a fundamental requirement is that they can be well dispersed and preferably also aligned in the composite, and they interact strongly with the surrounding matrix. On the other hand, a number of reports appeared suggesting doping liquid crystals with CNTs and this way to improve the properties of liquid crystals for electrooptic devices [47]. The incorporation of CNTs into organic or ceramic matrices (i.e. nanocomposites) and coating them with

*corresponding author; e-mail: jenikova@saske.sk functional materials (i.e. hybrids) has increased their applicability and so stimulated further interest. In this paper we study basic characteristic of CNTs to be able to create stable colloid system: liquid crystal and CNTs.

\section{Matherials and methods}

Commercially available carbon nanotubes (Sigma Aldrich) were used for our experiments. In our case they were the single walled CNTs (SWCNT) with diameter $d=1-4 \mathrm{~nm}$ and length $l=0.5-2 \mu \mathrm{m}$, and two kinds of multi walled CNTs (MWCNTs) with different diameter and lengths (long with $d=110-170 \mathrm{~nm}$ and $l=5-9 \mu \mathrm{m}$, and short with $d=5-40 \mathrm{~nm}$ and $l=0.5-2 \mu \mathrm{m})$. The Raman spectroscopy was performed on the spectrometer VERTEX80v (Bruker, Germany) at room temperature. Laser excitation wavelength was $1064 \mathrm{~nm}$. The diameter of the focused beam was approximately $100 \mu \mathrm{m}$. The laser was completely controlled by software and its power performance could be changed. Sample in powder form was placed in a small aluminium tube. Advantage is that it is enough to measure small amounts of samples. Pictures of all carbon nanotubes have been recorded using a high-resolution scanning-transmission electron microscope (TEM) JEOL 2100F with the Schottky field emission cathode and accelerating voltage $200 \mathrm{kV}$. In the standard mode, TEM has a point-to-point resolution 0.19. For magnetic measurements the samples were filled into gelatine capsules. Magnetic properties of all samples were measured by SQUID magnetometer (Quantum Design MPMS XL-5).

\section{Results and discussion}

The Raman spectroscopy is one of the most powerful techniques for the characterization of CNTs. It is also a convenient technique because it involves almost no sample preparation and leaves the material unharmed. There are four characteristic bands for CNTs. The band at $\approx 200 \mathrm{~cm}^{-1}$ is called radial breathing mode $(\mathrm{RBM})$ 
when all carbon atoms move in phases in the radial direction. It depends on the curvature and can be used to calculate a diameter of SWCNTs. The relatively broad $D$-band at $\approx 1340 \mathrm{~cm}^{-1}$ is assigned to $s p^{2}$-related defects and disorder in the graphitic structure of the material. The tangential $\mathrm{C}-\mathrm{C}$ stretching mode is located at $\approx 1560 \mathrm{~cm}^{-1}(G$-band $)$. The second order mode of the $D$-band ( $G^{\prime}$-band, also known as $2 D$-band) can be observed at $2450-2650 \mathrm{~cm}^{-1}$. The quantity of defects is often evaluated by comparing the intensity $I_{D} / I_{G}$ ratio of the $D$ - and $G$-bands [8]. The similar intensity of these two bands corresponds to a large number of structural failures. The most important aspect in the $G$-band is that we have different shapes according to the semiconducting or metallic character of CNTs, making it possible to easily distinguish between the two types. Figure 1 shows the Raman spectra of both MWCNTs as well as SWCNTs. From the ratio of the $D$ - and $G$-bands is clearly seen that both MWCNTs contain a large number of structural defects. On contrary, for SWCNTs the $D$-band is negligible comparing to the $G$-band.

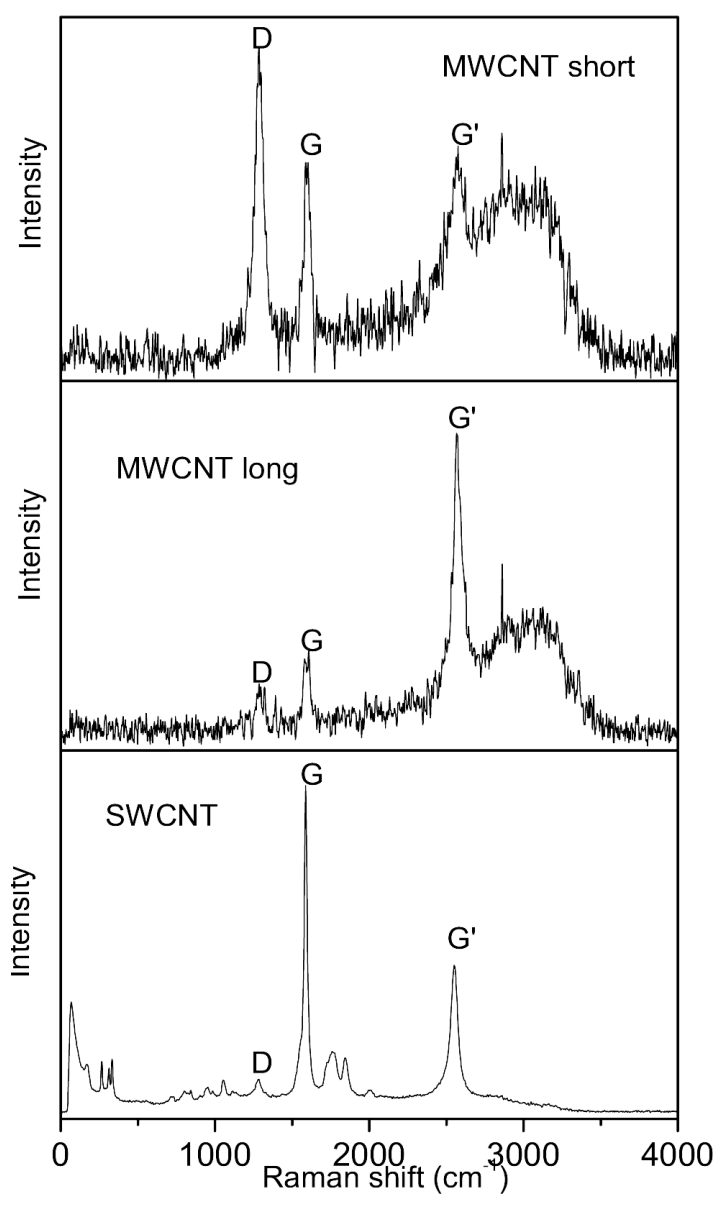

Fig. 1. Raman spectra of CNTs.

For TEM observations, the carbon nanotubes were dispersed in ultrasonic bath and fixed on carbon lacey film to avoid contrast losses by support film itself. In Fig. 2,

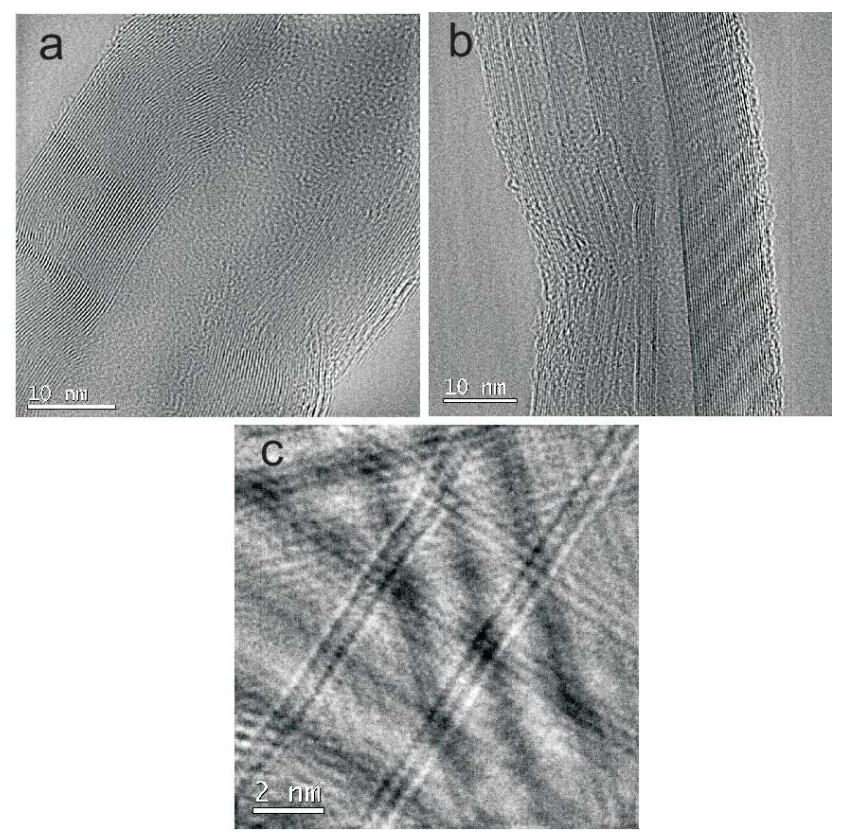

Fig. 2. HRTEM image of (a) short MWCNTs, (b) long MWCNTs and (c) SWCNTs.

series of TEM images of MWCNT and SWCNT taken in HRTEM mode are shown. In the first sample MWCNTs (Fig. 2a) have the various diameter. This value could not be determined accurately by TEM because of slightly warped walls and not uniform numbers of carbon layers contributing to wall thickness. Very similar result was obtained at second MWCNTs (Fig. 2b). However, it can be concluded that the average diameter of nanotubes was much lower. Figure $2 \mathrm{~b}$ represents the cranked and split wall sections typical for these MWCNTs. Although the CNT samples were dispersed before TEM observations, the SWCNTs were found only in aggregated form. For this reasons, together with extremely small TEM's depth of field, the only one SWCNT from nanotubes tangle could be imaged in-focus while the all others are out of focus. Moreover, because of phase-contrast nature of HRTEM imaging, the obtained images do not correspond to real sample structure exactly and suffer by scattering and interference effects. Due to this the walls of SWCNT (Fig. 2c) are imaged as three fringes instead of only one. Considering the middle bright fringe as one CNT wall the adjacent dark fringes on both side can be regarded as concomitant Fresnel fringes not representing structure.

The results obtained from Raman spectroscopy and TEM are in good agreement and confirmed the presence of large number of defects in long MWCNTs.

Figure 3 shows magnetization curves of both MWCNTs as wel as SWCNTs measured at $300 \mathrm{~K}$. It is seen that the short MWCNTs are diamagnetic while long MWCNTs and SWCNTs are ferromagnetic. In work [9], the magnetic properties of CNTs prepared by different synthesis techniques were studied. The authors showed that 


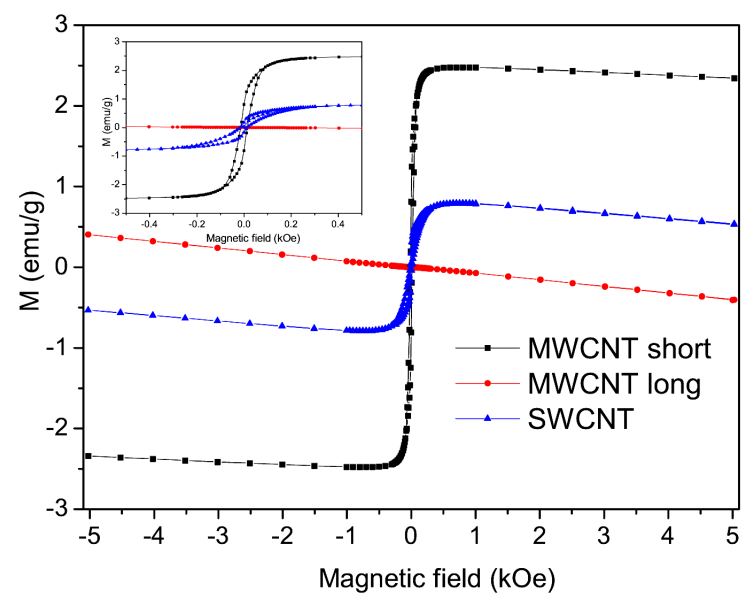

Fig. 3. Magnetization curves of short and long MWCNTs, and SWCNTs measured at $300 \mathrm{~K}$.

magnetic properties strongly depend on the used techniques and materials. Depending on this, it is possible to prepare ferromagnetic or diamagnetic CNTs. Our results are in good agreement with the results shown in [9]. We suppose the SWCNTs as well as MWCNTs are ferromagnetic due to presence of ferromagnetic impurities. However, due to presence of the impurities they are suitable for preparation of colloids, composed from liquid crystals and CNTs, sensitive to the magnetic field.

\section{Conclusions}

The obtained results showed that MWCNTs contain large amount of defects comparing to SWCNT. The results obtained from the Raman spectroscopy and TEM are in good agreement. Magnetic properties showed that long MWCNTs as well as SWCNTs are suitable to prepare colloids of liquid crystals and CNTs with higher sensitivity on the magnetic field. In our next investigation we will therefore focus on the preparation of stable colloids based on liquid crystal and ferromagnetic CNTs. It is now a widely-shared view that carbon-based materials are likely to be a major field in the new technologies.

\section{Acknowledgments}

This work was supported by projects VEGA 2/0045/13 and $1 / 0377 / 16$, the Slovak Research and Development Agency under the contract No. APVV-0171-10, Ministry of Education Agency for Structural Funds of EU in frame of project 26220120033 and 26220120021, and NanoCEXmat II 2622012035, and EU FP7 M-era.Net MACOSYS.

\section{References}

[1] J.P.F. Lagerwall, G. Scalia, Curr. Appl. Phys. 12, 1387 (2012).

[2] R. Saito, M.S. Dresselhaus, G. Dresselhaus, Physical Properties of Carbon Nanotubes, Imperial College Press, UK 1998.

[3] X. Blase, L.X. Benedict, E.L. Shirley, S.G. Louie, Phys. Rev. Lett. 72, 1878 (1994).

[4] H. Chen, W. Lee, N. Clark, Appl. Phys. Lett. 90, 033510 (2007).

[5] W. Lee, C. Wang, Y. Shih, Appl. Phys. Lett. 85, 513 (2004).

[6] C. Huang, C. Hu, H. Pan, K. Lo, Appl. Phys. Lett. 44, 8077 (2005).

[7] S.Y. Jeon, S.H. Shin, J.H. Lee, S.H. Lee, Y.H. Lee, Jpn. J. Appl. Phys. 46, 7801 (2007).

[8] M.S. Dresselhaus, A. Jorio, M. Hofmann, G. Dresselhaus, R. Saito, 0.1021/n1904286r Nano Lett. 10, 751 (2010).

[9] K. Lipert, M. Ritschel, A. Leonhardt, Y. Krupskaya, B. Buchner, R. Klingeler, J. Phys. Conf. Ser. 200, 072061 (2010). 